\title{
On products of multivalent close-to-star functions
}

Muhammad Arif', Jacek Dziok ${ }^{2 *}$, Mohsan Raza ${ }^{3}$ and Janusz Sokół ${ }^{4}$

${ }^{\text {*Correspondence: }}$

jdziok@univ.rzeszow.pl

${ }^{2}$ Faculty of Mathematics and Natural Sciences, University of Rzeszów, Rzeszów, 35-310, Poland Full list of author information is available at the end of the article

\begin{abstract}
In the present paper we define a class of products of multivalent close-to-star functions and determine the set of pairs $(|a|, r),|a|<r \leq 1-|a|$, such that every function from the class maps the $\operatorname{disk} \mathcal{D}(a, r):=\{z:|z-a|<r\}$ onto a domain starlike with respect to the origin. Some consequences of the obtained result are also considered.
\end{abstract}

MSC: 30C45; 30C50; 30C55

Keywords: analytic functions; close-to-star functions; generalized starlikeness; radius of starlikeness

\section{Introduction}

Let $\mathcal{A}$ denote the class of functions which are analytic in $\mathcal{D}=\mathcal{D}(0,1)$, where

$$
\mathcal{D}(a, r)=\{z:|z-a|<r\}
$$

and let $\mathcal{A}_{p}$ denote the class of functions $f \in \mathcal{A}$ of the form

$$
f(z)=z^{p}+\sum_{n=p+1}^{\infty} a_{n} z^{n} \quad\left(z \in \mathcal{D} ; p \in \mathbb{N}_{0}=\{0,1,2, \ldots\}\right) .
$$

A function $f \in \mathcal{A}_{p}$ is said to be starlike of order $\alpha$ in $\mathcal{D}(r):=\mathcal{D}(0, r)$ if

$$
\operatorname{Re}\left(\frac{z f^{\prime}(z)}{f(z)}\right)>\alpha \quad(z \in \mathcal{D}(r) ; 0 \leq \alpha<p) .
$$

A function $f \in \mathcal{A}_{1}$ is said to be convex of order $\alpha$ in $\mathcal{D}$ if

$$
\operatorname{Re}\left(1+\frac{z f^{\prime \prime}(z)}{f^{\prime}(z)}\right)>\alpha \quad(z \in \mathcal{D} ; 0 \leq \alpha<1) .
$$

We denote by $\mathcal{S}^{c}(\alpha)$ the class of all functions $f \in \mathcal{A}_{p}$, which are convex of order $\alpha$ in $\mathcal{D}$ and by $\mathcal{S}_{p}^{*}(\alpha)$ we denote the class of all functions $f \in \mathcal{A}_{p}$, which are starlike of order $\alpha$ in $\mathcal{D}$. We also set $\mathcal{S}^{*}(\alpha)=\mathcal{S}_{1}^{*}(0)$.

Let $\mathcal{H}$ be a subclass of the class $\mathcal{A}_{p}$. We define the radius of starlikeness of the class $\mathcal{H}$ by

$$
R^{*}(\mathcal{H})=\inf _{f \in \mathcal{H}}(\sup \{r \in(0,1]: f \text { is starlike of order } 0 \text { in } \mathcal{D}(r)\}) .
$$


We denote by $\mathcal{P}(\beta), 0<\beta \leq 1$, the class of functions $h \in \mathcal{A}$ such that $h(0)=1$ and

$$
h(\mathcal{D}) \subset \Pi_{\beta}:=\left\{w \in \mathbb{C} \backslash\{0\}:|\operatorname{Arg} w|<\beta \frac{\pi}{2}\right\},
$$

where $\operatorname{Arg} w$ denote the principal argument of the complex number $w$ (i.e. from the interval $(-\pi, \pi])$. The class $\mathcal{P}:=\mathcal{P}(1)$ is the well-known class of Carathéodory functions.

We say that a function $f \in \mathcal{A}$ belongs to the class $\mathcal{C S}_{p}^{*}(\alpha, \beta)$ if there exists a function $g \in \mathcal{S}_{p}^{*}(\alpha)$ such that

$$
\frac{f}{g} \in \mathcal{P}(\beta)
$$

In particular, we denote

$$
\mathcal{C S}_{p}^{*}(\alpha)=\mathcal{C S}_{p}^{*}(\alpha, 1), \quad \mathcal{C S}^{*}(\alpha)=\mathcal{C S}_{1}^{*}(\alpha), \quad \mathcal{C S}^{*}=\mathcal{C S}^{*}(0)
$$

The class $\mathcal{C S}^{*}$ is the well-known class of close-to-star functions with argument 0 .

Silverman [1] introduced the class of functions $F$ given by the formula

$$
F(z)=z \prod_{j=1}^{n}\left(\frac{f_{j}(z)}{z}\right)^{a_{j}} \prod_{j=1}^{n}\left(g_{j}^{\prime}(z)\right)^{b_{j}} \quad\left(f_{j} \in \mathcal{S}^{*}(\alpha), g_{j} \in \mathcal{S}^{c}(\beta)(j=1,2, \ldots, n)\right),
$$

where $a_{j}, b_{j}(j=1,2, \ldots, n)$ are positive real numbers satisfying the following conditions:

$$
\sum_{j=1}^{n} a_{j}=a, \quad \sum_{j=1}^{n} b_{j}=b
$$

Dimkov [2] studied the class of functions $F$ given by the formula

$$
F(z)=z \prod_{j=1}^{n}\left(\frac{f_{j}(z)}{z}\right)^{a_{j}} \quad\left(f_{j} \in \mathcal{S}^{*}\left(\alpha_{j}\right), j=1,2, \ldots, n\right)
$$

where $a_{j}(j=1,2, \ldots, n)$ are complex numbers satisfying the condition

$$
\sum_{j=1}^{n}\left(1-\alpha_{j}\right)\left|a_{j}\right| \leq a
$$

Let $p, n$ be positive integer and let $a, m, M, N$ be positive real numbers, $b \in[-m, m]$. Moreover, let

$$
\mathbf{a}=\left(a_{1}, a_{2}, \ldots, a_{n}\right), \quad \boldsymbol{\alpha}=\left(\alpha_{1}, \alpha_{2}, \ldots, \alpha_{n}\right), \quad \boldsymbol{\beta}=\left(\beta_{1}, \beta_{2}, \ldots, \beta_{n}\right)
$$

be fixed vectors, with

$$
a_{j} \in \mathbb{R}, \quad 0 \leq \alpha_{j}<p, \quad 0<\beta_{j} \leq 1 \quad(j=1,2, \ldots, n) .
$$


We denote by $\mathcal{H}_{p}^{n}(\mathbf{a}, \boldsymbol{\alpha}, \boldsymbol{\beta})$ the class of functions $F$ given by the formula

$$
F(z)=z^{p} \prod_{j=1}^{n}\left(\frac{f_{j}(z)}{z^{p}}\right)^{a_{j}} \quad\left(f_{j} \in \mathcal{C S}_{p}^{*}\left(\alpha_{j}, \beta_{j}\right), j=1, \ldots, n\right) .
$$

By $\mathcal{G}_{p}^{n}(m, b, c)$ we denote union of all classes $\mathcal{H}_{p}^{n}(\mathbf{a}, \boldsymbol{\alpha}, \boldsymbol{\beta})$ for which

$$
\sum_{j=1}^{n}\left(p-\alpha_{j}\right)\left|a_{j}\right|=m, \quad \sum_{j=1}^{n}\left(p-\alpha_{j}\right) \operatorname{Re} a_{j}=b, \quad \sum_{j=1}^{n} \beta_{j}\left|a_{j}\right|=c .
$$

Finally, let us denote

$$
\mathcal{G}_{p}^{n}(M, N):=\bigcup_{\substack{c \in[0, N] \\ m \in[0, M]}} \bigcup_{b \in[-m, m]} \mathcal{G}_{p}^{n}(m, b, c) .
$$

It is clear that the class $\mathcal{G}_{p}^{n}(M, N)$ contains functions $F$ given by the formula (2) for which

$$
\sum_{j=1}^{n}\left(p-\alpha_{j}\right)\left|a_{j}\right| \leq M, \quad \sum_{j=1}^{n} \beta_{j}\left|a_{j}\right| \leq N .
$$

Aleksandrov [3] stated and solved the following problem.

Problem 1 Let $\mathcal{H}$ be the class of functions $f \in \mathcal{A}$ that are univalent in $\mathcal{D}$ and let $\Delta \subset \mathcal{D}$ be a domain starlike with respect to an inner point $\omega$ with smooth boundary given by the formula

$$
z(t)=\omega+r(t) e^{i t} \quad(0 \leq t \leq 2 \pi) .
$$

Find conditions for the function $r(t)$ such that for each $f \in \mathcal{H}$ the image domain $f(\Delta)$ is starlike with respect to $f(\omega)$.

Świtoniak and Stankiewicz [4, 5], Dimkov and Dziok [6] (see also [7]) have investigated a similar problem of generalized starlikeness.

Problem 2 Let $\mathcal{H} \subset \mathcal{A}$. Determine the set $B^{*}(\mathcal{H})$ of all pairs $(a, r) \in \mathcal{D} \times \mathbb{R}$, such that

$$
|a|<r \leq 1-|a|,
$$

and every function $f \in \mathcal{H}$ maps the disk $\mathcal{D}(a, r)$ onto a domain starlike with respect to the origin. The set $B^{*}(\mathcal{H})$ is called the set of generalized starlikeness of the class $\mathcal{H}$.

We note that

$$
R^{*}(\mathcal{H})=\sup \left\{r:(0, r) \in B^{*}(\mathcal{H})\right\} .
$$

In this paper we determine the sets $B^{*}\left(\mathcal{H}_{p}^{n}(\mathbf{a}, \boldsymbol{\alpha}, \boldsymbol{\beta})\right), B^{*}\left(\mathcal{G}_{p}^{n}(m, b, c)\right)$ and $B^{*}\left(\mathcal{G}_{p}^{n}(M, N)\right)$. The sets of generalized starlikeness for some subclasses of the defined classes are also considered. Moreover, we obtain the radii of starlikeness of these classes of functions. 


\section{Main results}

We start from listing some lemmas which will be useful later on.

Lemma 1 [5] A function $f \in \mathcal{A}$ maps the disk $\mathcal{D}(a, r),|a|<r \leq 1-|a|$, onto a domain starlike with respect to the origin if and only if

$$
\operatorname{Re} \frac{e^{i \theta} f^{\prime}\left(a+r e^{i \theta}\right)}{f\left(a+r e^{i \theta}\right)} \geq 0 \quad(0 \leq \theta \leq 2 \pi) .
$$

For a function $f \in \mathcal{S}_{p}^{*}(\alpha)$ it is easy to verify that

$$
\left|\frac{z f^{\prime}(z)}{f(z)}-\alpha-(p-\alpha) \frac{1+|z|^{2}}{1-|z|^{2}}\right| \leq \frac{2(p-\alpha)|z|}{1-|z|^{2}} \quad(z \in \mathcal{D}) .
$$

Thus, after some calculations we get the following lemma.

Lemma 2 Let $f \in \mathcal{S}_{p}^{*}(\alpha), a, \theta \in \mathbb{R}, z \in \mathcal{D}_{0}:=\mathcal{D} \backslash\{0\}$. Then

$$
\operatorname{Re}\left[a e^{i \theta}\left(\frac{f^{\prime}(z)}{f(z)}-\frac{p}{z}\right)\right] \geq \frac{2(p-\alpha)}{1-|z|^{2}} \operatorname{Re}\left(a \bar{z} e^{i \theta}-|a|\right) .
$$

Lemma 3 [8] If $h \in \mathcal{P}(\beta)$, then

$$
\left|\frac{h^{\prime}(z)}{h(z)}\right| \leq \frac{2 \beta}{1-|z|^{2}} \quad(z \in \mathcal{D}) \text {. }
$$

Theorem 1 Let $m, b, c$ be defined by (3) and set

$$
\begin{aligned}
\mathcal{B}^{\prime} & =\left\{(a, r) \in \mathbb{C} \times \mathbb{R}:\left\{\begin{array}{l}
0 \leq r \leq r_{1},|a|<r, \\
r_{1}<r<r_{2},|a| \leq \varphi(r), \\
r_{2} \leq r<q,|a| \leq q-r
\end{array}\right\}\right\}, \\
\mathcal{B}^{\prime \prime} & =\{(a, r) \in \mathbb{C} \times \mathbb{R}:|a|<r \leq q-|a|\},
\end{aligned}
$$

where

$$
\begin{aligned}
& r_{1}=\frac{p}{4(m+c)}, \\
& r_{2}=\frac{p(m+c)}{\left(m+c+\sqrt{(m+c)^{2}-2 b p+p^{2}}\right)^{2}} \\
& q=\frac{p}{m+c+\sqrt{(m+c)^{2}-2 b p+p^{2}}}, \\
& \varphi(r)=\sqrt{r^{2}-\frac{(1-2 \sqrt{r(m+c)})^{2}}{2 b-1}} .
\end{aligned}
$$

Moreover, set

$$
\mathcal{B}= \begin{cases}\mathcal{B}^{\prime} & \text { for } b>p / 2, \\ \mathcal{B}^{\prime \prime} & \text { for } b \leq p / 2 .\end{cases}
$$


If $(a, r) \in \mathcal{B}$, then a function $F \in \mathcal{H}_{p}^{n}(\mathbf{a}, \boldsymbol{\alpha}, \boldsymbol{\beta})$ maps the disk $\mathcal{D}(a, r)$ onto a domain starlike with respect to the origin. The result is sharp for $b \leq p / 2$ and for $b>p / 2$ the set $\mathcal{B}$ cannot be larger than $\mathcal{B}^{\prime \prime}$. It means that

$$
\begin{aligned}
& \mathcal{B}^{\prime} \subset B^{*}\left(\mathcal{H}_{p}^{n}(\mathbf{a}, \boldsymbol{\alpha}, \boldsymbol{\beta})\right) \subset \mathcal{B}^{\prime \prime} \quad(b>p / 2), \\
& B^{*}\left(\mathcal{H}_{p}^{n}(\mathbf{a}, \boldsymbol{\alpha}, \boldsymbol{\beta})\right)=\mathcal{B}^{\prime \prime} \quad(b \leq p / 2) .
\end{aligned}
$$

Proof Let $F$ belong to the class $\mathcal{H}_{p}^{n}(\mathbf{a}, \boldsymbol{\alpha}, \boldsymbol{\beta})$ and let $z=a+r e^{i \theta} \in \mathcal{D}$ satisfy (5). The functions

$$
g_{j, s}(z)=e^{-i s} f_{j}\left(e^{i s} z\right) \quad(z \in \mathcal{D} ; j=1,2, \ldots, n, s \in \mathbb{R})
$$

belong to the class $\mathcal{C S}_{p}^{*}\left(\alpha_{j}, \beta_{j}\right)$ together with the functions $f_{j}(z)$. Thus, by (2) the functions

$$
G_{s}(z)=e^{-i s} F\left(e^{i s} z\right) \quad(z \in \mathcal{D} ; s \in \mathbb{R})
$$

belong to the class $\mathcal{H}_{p}^{n}(\mathbf{a}, \boldsymbol{\alpha}, \boldsymbol{\beta})$ together with the function $F(z)$. In consequence, we have

$$
(a, r) \in B^{*}\left(\mathcal{H}_{p}^{n}(\mathbf{a}, \boldsymbol{\alpha}, \boldsymbol{\beta})\right) \quad \Longleftrightarrow \quad(|a|, r) \in B^{*}\left(\mathcal{H}_{p}^{n}(\mathbf{a}, \boldsymbol{\alpha}, \boldsymbol{\beta})\right) \quad(a \in \mathcal{D}, r \geq 0) .
$$

Therefore, without loss of generality we may assume that $a$ is nonnegative real number. Since $f_{j} \in \mathcal{C} \mathcal{S}_{p}^{*}\left(\alpha_{j}, \beta_{j}\right)$, there exist functions $g_{j} \in \mathcal{S}_{p}^{*}\left(\alpha_{j}\right)$ and $h_{j} \in \mathcal{P}\left(\beta_{j}\right)$ such that

$$
\frac{f_{j}(z)}{g_{j}(z)}=h_{j}(z) \quad(z \in \mathcal{D})
$$

or equivalently

$$
f_{j}(z)=g_{j}(z) h_{j}(z) \quad(z \in \mathcal{D})
$$

After logarithmic differentiation of the equality (2) we obtain

$$
\frac{F^{\prime}(z)}{F(z)}=\frac{p}{z}+\sum_{j=1}^{n} a_{j}\left(\frac{f_{j}^{\prime}(z)}{f_{j}(z)}-\frac{p}{z}\right) \quad(z \in \mathcal{D}) .
$$

Thus, using (18) we have

$$
\begin{aligned}
\operatorname{Re} \frac{e^{i \theta} F^{\prime}(z)}{F(z)} & =\operatorname{Re} \frac{p e^{i \theta}}{z}+\sum_{j=1}^{n} \operatorname{Re}\left(a_{j} e^{i \theta}\left(\frac{g_{j}^{\prime}(z)}{g_{j}(z)}-\frac{p}{z}\right)\right)+\sum_{j=1}^{n} \operatorname{Re}\left(a_{j} e^{i \theta} \frac{h_{j}^{\prime}(z)}{h_{j}(z)}\right) \\
& \geq \operatorname{Re} \frac{p e^{i \theta}}{z}+\sum_{j=1}^{n} \operatorname{Re}\left(a_{j} e^{i \theta}\left(\frac{g^{\prime}(z)}{g_{j}(z)}-\frac{p}{z}\right)\right)-\sum_{j=1}^{n}\left|a_{j}\right|\left|\frac{h_{j}^{\prime}(z)}{h_{j}(z)}\right|
\end{aligned}
$$

By Lemma 2 and Lemma 3 we obtain

$$
\begin{aligned}
\operatorname{Re} \frac{e^{i \theta} F^{\prime}(z)}{F(z)} \geq & \operatorname{Re} \frac{p e^{i \theta}}{z}+\frac{2}{1-|z|^{2}} \sum_{j=1}^{n}\left(p-\alpha_{j}\right) a_{j} \operatorname{Re}\left(\bar{z} e^{i \theta}\right) \\
& -\frac{2}{1-|z|^{2}} \sum_{j=1}^{n}\left(p-\alpha_{j}\right)\left|a_{j}\right|-\frac{2}{1-|z|^{2}} \sum_{j=1}^{n}\left|a_{j}\right| \beta_{j} .
\end{aligned}
$$


Setting $z=a+r e^{i \theta}$ and using (3) the above inequality yields

$$
\operatorname{Re} \frac{e^{i \theta} F^{\prime}\left(a+r e^{i \theta}\right)}{F\left(a+r e^{i \theta}\right)} \geq \operatorname{Re} \frac{p e^{i \theta}}{a+r e^{i \theta}}+2 \frac{\operatorname{Re}\left(r+a e^{-i \theta}\right) b-m-c}{1-\left|a+r e^{i \theta}\right|^{2}} .
$$

By Lemma 1 it is sufficient to show that the right-hand side of the last inequality is nonnegative, that is,

$$
\operatorname{Re} \frac{p}{r+a e^{-i \theta}}+2 \frac{\operatorname{Re}\left(r+a e^{-i \theta}\right) b-m-c}{1-\left|r+a e^{-i \theta}\right|^{2}} \geq 0 .
$$

If we put

$$
r+a e^{-i \theta}=x+y i
$$

then we obtain

$$
\frac{p x}{x^{2}+y^{2}}+2 \frac{b x-m-c}{1-x^{2}-y^{2}} \geq 0 .
$$

Thus, using the equality

$$
(x-r)^{2}+y^{2}=a^{2},
$$

we obtain

$$
\begin{aligned}
w(x)= & 2 r(2 b-p) x^{2}-\left((2 b-p)\left(r^{2}-a^{2}\right)+4 r(m+c)-p\right) x \\
& +2(m+c)\left(r^{2}-a^{2}\right) \geq 0 .
\end{aligned}
$$

The discriminant $\Delta$ of $w(x)$ is given by

$$
\begin{aligned}
\Delta= & \left((2 b-p)\left(r^{2}-a^{2}\right)+4 r(m+c)-p\right)^{2} \\
& -16 r(2 b-p)(m+c)\left(r^{2}-a^{2}\right)=A_{1} A_{2},
\end{aligned}
$$

where

$$
\begin{aligned}
& A_{1}=(p-2 b)\left(r^{2}-a^{2}\right)+p+4 r(m+c)+4 \sqrt{r p(m+c)}, \\
& A_{2}=(p-2 b)\left(r^{2}-a^{2}\right)+p+4 r(m+c)-4 \sqrt{r p(m+c)} .
\end{aligned}
$$

Let

$$
D=\left\{(a, r) \in \mathbb{R}^{2}: 0 \leq a<r \leq 1-a\right\} .
$$

First, we discuss the case $b>p / 2$. Thus, the inequality (21) is satisfied for every $x \in[r-$ $a, r+a]$ if one of the following conditions is fulfilled:

$1^{\circ} \Delta \leq 0$,

$2^{\circ} \quad \Delta>0, w(r-a) \geq 0$ and $x_{0} \leq r-a$,

$3^{\circ} \Delta>0, w(r+a) \geq 0$ and $x_{0} \geq r+a$, 
where

$$
x_{0}=\frac{(2 b-p)\left(r^{2}-a^{2}\right)+4 r(m+c)-p}{4(2 b-p) r} .
$$

Ad $1^{\circ}$. Since $A_{1}>0$, by (22), the condition $\Delta \leq 0$ is equivalent to the inequality $A_{2} \leq 0$. Then

$$
\mathcal{B}_{1}:=\{(a, r) \in D: \Delta \leq 0\}=\left\{(a, r) \in D: A_{2} \leq 0\right\}=\{(a, r) \in D: a \leq \varphi(r)\},
$$

where $\varphi$ is defined by (13). Let

$$
\gamma=\{(a, r) \in \bar{D}: a=\varphi(r)\} .
$$

Then $\gamma$ is the curve which is tangent to the straight lines $a=r$ and $a=q-r$ at the points

$$
S_{1}=\left(r_{1}, r_{1}\right) \text { and } S_{2}=\left(q-r_{2}, r_{2}\right) \text {, }
$$

where $r_{1}, r_{2}, q$ are defined by (10), (11), (12), respectively.

Moreover, $\gamma$ cuts the straight line $a=0$ at the points

$$
\begin{aligned}
& r_{3}=p(\sqrt{m+c+\sqrt{p(2 b-p)}}+\sqrt{m+c})^{-2}, \\
& r_{4}=p(\sqrt{m+c-\sqrt{p(2 b-p)}}+\sqrt{m+c})^{-2} .
\end{aligned}
$$

Since

$$
0<r_{3}<r_{1}<r_{2}<r_{4}<q
$$

we have

$$
\gamma=\left\{(a, r) \in \mathbb{R}^{2}: r_{3} \leq r \leq r_{4}, a=\varphi(r)\right\},
$$

and consequently

$$
\mathcal{B}_{1}=\left\{(a, r) \in \mathbb{R}^{2}: r_{3} \leq r \leq r_{4}, 0 \leq a \leq \varphi(r)\right\},
$$

where $\varphi$ is defined by (13) (see Figure 1).

Ad $2^{\circ}$. Let

$$
\mathcal{B}_{2}:=\left\{(a, r) \in D: \Delta>0 \wedge w(r-a) \geq 0 \wedge x_{0} \leq r-a\right\} .
$$

It is easy to verify that

$$
\begin{aligned}
w(r-a) & =(r-a)\left((2 b-1)(r-a)^{2}-2(m+c)(r-a)+1\right) \\
& =(2 b-1)(r-a)\left(r-a-q^{\prime}\right)(r-a-q),
\end{aligned}
$$




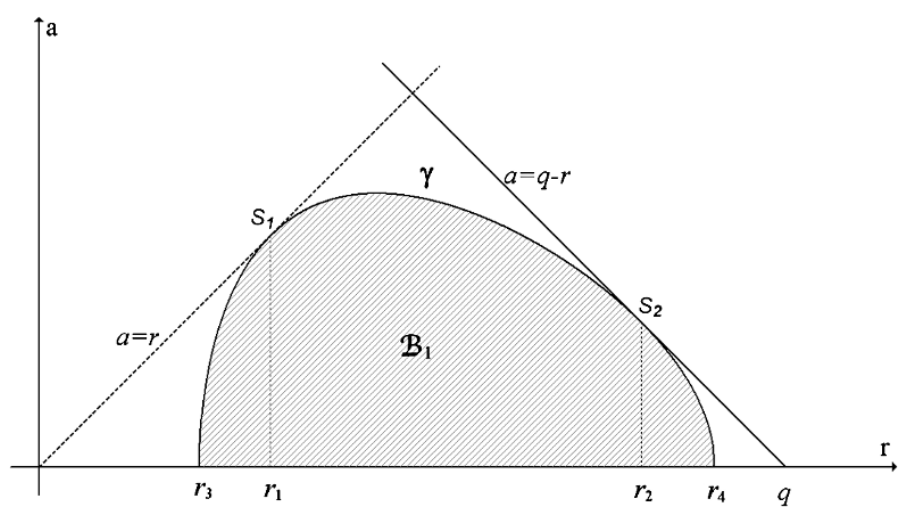

Figure 1 The set $\mathcal{B}_{1}$.

where $q$ is defined by (12) and

$$
q^{\prime}=p\left(m+c-\sqrt{(m+c)^{2}-2 b p+p^{2}}\right)^{-1} .
$$

Since

$$
0<q<1<q^{\prime} \quad(p / 2<b \leq m,(a, r) \in D),
$$

we see that

$$
(r-a)\left(r-a-q^{\prime}\right)<0 \quad((a, r) \in D) .
$$

Thus, the inequality $w(r-a) \geq 0$ is true if $a \geq r-q$. The inequality $x_{0} \leq r-a$ may be written in the form

$$
(2 b-p) a^{2}+3(2 b-p) r^{2}-4(m+c) r-4(2 b-p) a r+p \geq 0 .
$$

The hyperbola $h_{1}$, which is the boundary of the set of all pairs $(a, r) \in \mathbb{R}^{2}$ satisfying (31), cuts the boundary of the set $D$ at the point $S_{1}$ defined by (27) and at the point $\left(r_{5}, 0\right)$, where

$$
r_{5}=p\left(2(m+c)+\sqrt{4(m+c)^{2}-3 p(2 b-p)}\right)^{-1} .
$$

It is easy to verify that

$$
r_{3}<r_{5}<r_{4}<q .
$$

Thus we determine the set

$$
\mathcal{B}_{2}=\left\{(a, r) \in \mathbb{R}^{2}:\left\{\begin{array}{l}
0 \leq r \leq r_{3}, 0 \leq a<r, \\
r_{3}<r<r_{1}, \varphi(r)<a<r
\end{array}\right\}\right\},
$$

where $\varphi$ is defined by (13) (see Figure 2). 


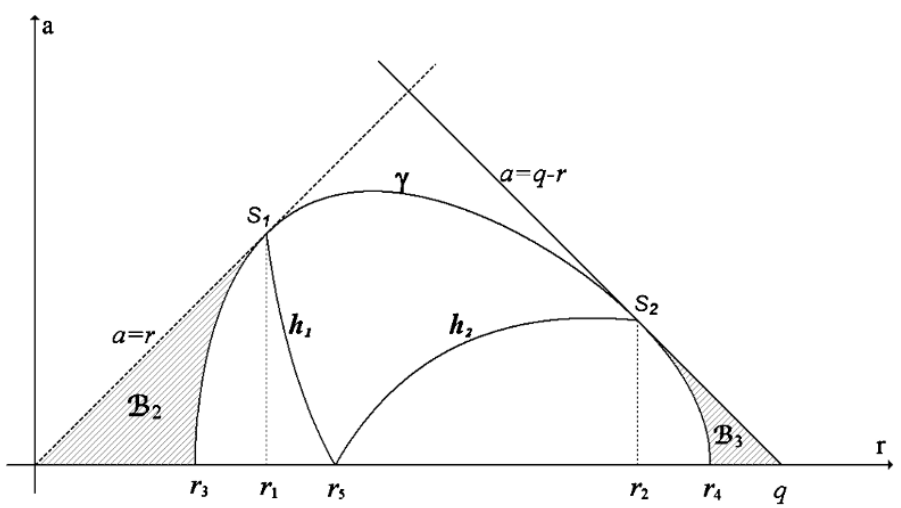

Figure 2 The sets $\mathcal{B}_{2}$ and $\mathcal{B}_{3}$.

Ad $3^{\circ}$. Let

$$
\mathcal{B}_{3}:=\left\{(a, r) \in D: \Delta>0 \wedge w(r+a) \geq 0 \wedge x_{0} \geq r+a\right\}
$$

and let $q$ and $q^{\prime}$ be defined by (12) and (29), respectively. Then

$$
\begin{aligned}
w(a+r) & =(r+a)\left[(2 b-p)(r+a)^{2}-2(m+c)(r+a)+p\right] \\
& =(2 b-p)(r+a)\left(r+a-q^{\prime}\right)(r+a-q) .
\end{aligned}
$$

Moreover, by (30) we have

$$
(r+a)\left(r+a-q^{\prime}\right)<0 \quad((a, r) \in D) .
$$

Thus, we conclude that the inequality $w(r+a) \geq 0$ is true if $a \leq q-r$. The inequality $x_{0} \geq r+a$ may be written in the form

$$
(2 b-p) a^{2}+3(2 b-p) r^{2}-4(m+c) r+4(2 b-p) a r+p \leq 0 .
$$

The hyperbola $h_{2}$, which is the boundary of the set of all pairs $(a, r) \in \mathbb{R}^{2}$ satisfying (34), cuts the boundary of the set $D$ at the point $S_{2}$ defined by $(27)$ and at the point $\left(r_{5}, 0\right)$, where $r_{5}$ is defined by (32). Thus, we describe the set

$$
\mathcal{B}_{3}=\left\{(a, r) \in \mathbb{R}^{2}:\left\{\begin{array}{l}
r_{2}<r<r_{4}, \varphi(r)<a \leq q-r, \\
r_{4}<r<q, 0 \leq a \leq q-r
\end{array}\right\}\right\},
$$

where $\varphi$ is defined by (13) (see Figure 2). The union of the sets $\mathcal{B}_{1}, \mathcal{B}_{2}, \mathcal{B}_{3}$ defined by (28), (33), and (35) gives the set

$$
\widetilde{\mathcal{B}}^{\prime}=\left\{(a, r) \in \mathbb{R}^{2}:\left\{\begin{array}{l}
0 \leq r \leq r_{1}, 0 \leq a<r \\
r_{1}<r<r_{2}, 0 \leq a \leq \varphi(r), \\
r_{2} \leq r<q, 0 \leq a \leq q-r
\end{array}\right\}\right\}
$$


Thus, by (17) we have

$$
\mathcal{B}^{\prime} \subset B^{*}\left(\mathcal{H}_{p}^{n}(\mathbf{a}, \boldsymbol{\alpha}, \boldsymbol{\beta})\right) \quad(b>p / 2),
$$

where $\mathcal{B}^{\prime}$ is defined by (8).

Now, let $b<p / 2$. Then the inequality (21) is satisfied for every $x \in[r-a, r+a]$ if

$$
w(r-a) \geq 0 \quad \text { and } \quad w(r+a) \geq 0 .
$$

We see that

$$
\begin{aligned}
& w(a+r)=(2 b-p)(r+a)\left(r+a-q^{\prime}\right)(r+a-q), \\
& w(r-a)=(2 b-p)(r-a)\left(r-a-q^{\prime}\right)(r-a-q),
\end{aligned}
$$

where $q$ and $q^{\prime}$ are defined by (12) and (29), respectively. Since

$$
q^{\prime}<0<q<1 \quad(b<p / 2),
$$

the condition (37) is satisfied if $(a, r) \in D$ and

$$
a \leq q-r
$$

Let $b=1 / 2$. Then by (21) we obtain

$$
(p-4 r(m+c)) x+2(m+c)\left(r^{2}-a^{2}\right) \geq 0 .
$$

The above inequality holds for every $x \in[r-a, r+a]$ if $(a, r) \in D$ and

$$
r-a \leq \frac{p}{2(m+c)}
$$

or equivalently (38). Thus, by (17) we have

$$
\mathcal{B}^{\prime \prime} \subset B^{*}\left(\mathcal{H}_{p}^{n}(\mathbf{a}, \boldsymbol{\alpha}, \boldsymbol{\beta})\right) \quad(b \leq p / 2)
$$

where $\mathcal{B}^{\prime \prime}$ is defined by (9). Because the function

$$
F(z)=z^{p} \prod_{1}^{n}\left(\frac{1}{\left(1+\operatorname{sgn}\left(a_{j}\right) z\right)^{2(p-\alpha)}}\left(\frac{1-z}{1+z}\right)^{\beta_{j} \operatorname{sgn}\left(a_{j}\right)}\right)^{a_{j}} \quad(z \in \mathcal{D})
$$

belongs to the class $\mathcal{H}_{p}^{n}(\mathbf{a}, \boldsymbol{\alpha}, \boldsymbol{\beta})$, and for $z=a+r, \theta=0, a+r>q$ we have

$$
\operatorname{Re} \frac{e^{i \theta} F^{\prime}(z)}{F(z)}=\frac{p-2(m+c)(a+r)+(2 b-p)(a+r)^{2}}{(a+r)\left(1-(a+r)^{2}\right)}<0 .
$$

Lemma 1 yields

$$
B^{*}\left(\mathcal{H}_{p}^{n}(\mathbf{a}, \boldsymbol{\alpha}, \boldsymbol{\beta})\right) \subset \mathcal{B}^{\prime \prime}
$$


From (36) and (41) we have (15), while (39) and (41) give (16), which completes the proof.

Since the set $\mathcal{B}$ defined by (14) is dependent only of $m, b, c$, the following result is an immediate consequence of Theorem 1 .

Theorem 2 Let $\mathcal{B}$ be defined by (14). If $(a, r) \in \mathcal{B}$, then a function $F \in \mathcal{G}_{p}^{n}(m, b, c)$ maps the disk $\mathcal{D}(a, r)$ onto a domain starlike with respect to the origin. The obtained result is sharp for $b \leq p / 2$ and for $b>p / 2$ the set $\mathcal{B}$ cannot be larger than $\mathcal{B}^{\prime \prime}$, where $\mathcal{B}^{\prime \prime}$ is defined by (8). It means that

$$
\begin{aligned}
& \mathcal{B} \subset B^{*}\left(\mathcal{G}_{p}^{n}(m, b, c)\right) \subset \mathcal{B}^{\prime \prime} \quad(b>p / 2), \\
& B^{*}\left(\mathcal{G}_{p}^{n}(m, b, c)\right)=\mathcal{B} \quad(b \leq p / 2) .
\end{aligned}
$$

The functions described by (40), with (3) are the extremal functions.

\section{Theorem 3}

$$
B^{*}\left(\mathcal{G}^{n}(M, N)\right)=\left\{(a, r) \in \mathbb{C} \times \mathbb{R}:|a|<r \leq q_{1}-|a|\right\},
$$

where

$$
q_{1}=\frac{p}{M+N+\sqrt{(M+N)^{2}+2 M p+p^{2}}} .
$$

The equality in (42) is realized by the function $F$ of the form

$$
F(z)=z^{p} \frac{(1-z)^{2 M+N}}{(1+z)^{N}} \quad(z \in \mathcal{D}) .
$$

Proof Let $M, N$ be positive real numbers and let $\mathcal{B}^{\prime}=\mathcal{B}^{\prime}(m, b, c), \mathcal{B}^{\prime \prime}=\mathcal{B}^{\prime \prime}(m, b, c), q=$ $q(m, b, c)$ and $\varphi(r)=\varphi(r ; m, b, c)$ be defined by (8), (9), (12), and (13), respectively.

It is easy to verify that

$$
\varphi(r ; m, b, c) \geq q(m, p / 2, c)-r \quad(1 /(2 q(m, p / 2, c)) \leq r \leq q(m, p / 2, c), p / 2<b \leq m) .
$$

Moreover, the function $q=q(m, b, c)$ is decreasing with respect to $m$ and $c$, and increasing with respect to $b$. Thus, from Theorems 1 and 2 we have (see Figure 3)

$$
\begin{aligned}
& B^{*}\left(\mathcal{G}^{n}(m, p / 2, c)\right)=\mathcal{B}^{\prime \prime}(m, p / 2, c) \subset \mathcal{B}^{\prime}(m, b, c) \subset B^{*}\left(\mathcal{G}^{n}(m, b, c)\right) \\
& \quad(m \in[0, M], c \in[0, N], b \in(p / 2, m])
\end{aligned}
$$

and

$$
\begin{gathered}
B^{*}\left(\mathcal{G}^{n}(M,-M, N)\right) \subset B^{*}\left(\mathcal{G}^{n}(m, b, c)\right) \subset B^{*}\left(\mathcal{G}^{n}(m, p / 2, c)\right) \\
(m \in[0, M], c \in[0, N], b \in[-m, p / 2]) .
\end{gathered}
$$

Therefore, by (4) we obtain

$$
B^{*}\left(\mathcal{G}^{n}(M, N)\right)=B^{*}\left(\mathcal{G}^{n}(M,-M, N)\right)
$$




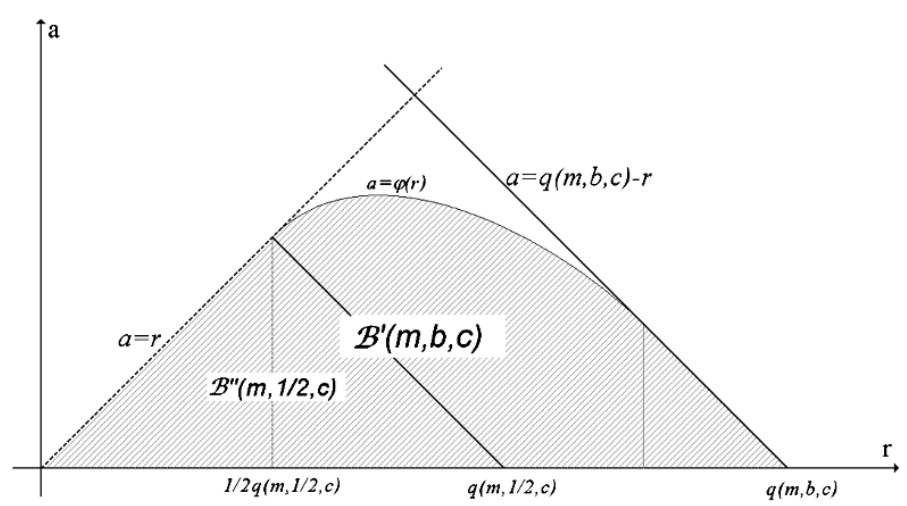

Figure 3 The sets $\mathcal{B}^{\prime}$ and $\mathcal{B}^{\prime \prime}$.

and by Theorem 2 we get (42). Putting $m=M, b=-M$ in (3) we see that $a_{1}, a_{2}, \ldots, a_{n}$ are negative real numbers. Thus, the extremal function (40) has the form

$$
F(z)=z^{p} \prod_{j=1}^{n}\left(\frac{1}{(1-z)^{2\left(1-\alpha_{j}\right)}}\left(\frac{1+z}{1-z}\right)^{\beta_{j}}\right)^{a_{j}} \quad(z \in \mathcal{D})
$$

or equivalently

$$
F(z)=\frac{z^{p}}{(1-z)^{-2 \sum_{j=1}^{n}\left(1-\alpha_{j}\right)\left|a_{j}\right|}}\left(\frac{1+z}{1-z}\right)^{-\sum_{j=1}^{n} \beta_{j}\left|a_{j}\right|} \quad(z \in \mathcal{D}) .
$$

Consequently, using (3) we obtain

$$
F(z)=\frac{z^{p}}{(1-z)^{-2 M}}\left(\frac{1+z}{1-z}\right)^{-N} \quad(z \in \mathcal{D}),
$$

that is, we have the function (43) and the proof is completed.

Since $\mathcal{H}_{p}^{a}((1),(\alpha),(\beta))=\mathcal{C S}_{p}^{*}(\alpha, \beta)$, by Theorem 1 we obtain the following theorem.

Theorem 4 Let $0 \leq \alpha<p, 0<\beta \leq 1$, and

$$
\begin{aligned}
\mathcal{B}^{\prime} & =\left\{(a, r) \in \mathbb{C} \times \mathbb{R}:\left\{\begin{array}{l}
0 \leq r \leq r_{1},|a|<r, \\
r_{1}<r<r_{2},|a| \leq \varphi(r), \\
r_{2} \leq r<q,|a| \leq q-r
\end{array}\right\}\right\}, \\
\mathcal{B}^{\prime \prime} & =\{(a, r) \in \mathbb{C} \times \mathbb{R}:|a|<r \leq q-|a|\},
\end{aligned}
$$

where

$$
\begin{aligned}
& r_{1}=\frac{1}{4(\beta-\alpha+p)}, \\
& r_{2}=\frac{p(\beta+p-\alpha)}{\left(\beta+p-\alpha+\sqrt{(\beta-\alpha)^{2}+2 \beta p}\right)^{2}}
\end{aligned}
$$




$$
\begin{aligned}
& q=\frac{p}{\beta+p-\alpha+\sqrt{(\beta-\alpha)^{2}+2 \beta p}}, \\
& \varphi(r)=\sqrt{r^{2}-\frac{(1-2 \sqrt{r(\beta-\alpha+p)})^{2}}{2 p-2 \alpha-1}} .
\end{aligned}
$$

Moreover, let us put

$$
\mathcal{B}= \begin{cases}\mathcal{B}^{\prime} & \text { for } \alpha<p / 2 \\ \mathcal{B}^{\prime \prime} & \text { for } \alpha \geq p / 2\end{cases}
$$

If $(|a|, r) \in \mathcal{B}$, then the function $f \in \mathcal{C S}_{p}^{*}(\alpha, \beta)$ maps the disk $\mathcal{D}(a, r)$ onto a domain starlike with respect to the origin. The obtained result is sharp for $\alpha \geq p / 2$ and for $\alpha<p / 2$ the set $\mathcal{B}$ cannot be larger then $\mathcal{B}^{\prime \prime}$. It means that

$$
\begin{aligned}
& \mathcal{B} \subset B^{*}\left(\mathcal{C S}_{p}^{*}(\alpha)\right) \subset \mathcal{B}^{\prime \prime} \quad(\alpha<p / 2), \\
& B^{*}\left(\mathcal{C S}_{p}^{*}(\alpha)\right)=\mathcal{B} \quad(\alpha \geq p / 2) .
\end{aligned}
$$

The function $F$ of the form

$$
F(z)=z^{p} \frac{(1+z)^{\beta}}{(1-z)^{2 p-2 \alpha+\beta}} \quad(z \in \mathcal{D})
$$

is the extremal function.

Using (6) and Theorems 1-4, we obtain the radii of starlikeness for the classes $\mathcal{H}_{p}^{n}(\mathbf{a}, \boldsymbol{\alpha}, \boldsymbol{\beta})$, $\mathcal{G}_{p}^{n}(m, b, c), \mathcal{G}_{p}^{n}(M, N), \mathcal{C S}_{p}^{*}(\alpha, \beta)$.

Corollary 1 The radius of starlikeness of the classes $\mathcal{G}_{p}^{n}(m, b, c)$ and $\mathcal{H}_{p}^{n}(\mathbf{a}, \boldsymbol{\alpha}, \boldsymbol{\beta})$ is given by

$$
R^{*}\left(\mathcal{G}_{p}^{n}(m, b, c)\right)=R^{*}\left(\mathcal{H}_{p}^{n}(\mathbf{a}, \boldsymbol{\alpha}, \boldsymbol{\beta})\right)=\frac{p}{m+c+\sqrt{(m+c)^{2}-2 b p+p^{2}}} .
$$

Corollary 2 The radius of starlikeness of the class $\mathcal{G}_{p}^{n}(M, N)$ is given by

$$
R^{*}\left(\mathcal{G}_{p}^{n}(M, N)\right)=\frac{p}{M+N+\sqrt{(M+N)^{2}+2 M p+p^{2}}} .
$$

Corollary 3 The radius of starlikeness of the class $\mathcal{C S}_{p}^{*}(\alpha, \beta)$ is given by

$$
R^{*}\left(\mathcal{C S}_{p}^{*}(\alpha, \beta)\right)=\frac{p}{\beta+p-\alpha+\sqrt{(\beta-\alpha)^{2}+2 \beta p}} .
$$

Remark 1 Putting $\beta=1$ in Corollary 3 we get the radius of starlikeness of the class $\mathcal{C S}_{p}^{*}(\alpha)=\mathcal{C S}_{p}^{*}(\alpha, 1)$ obtained by Dziok [7]. Putting $p=\beta=1$ we get the radius of starlikeness of the class $\mathcal{C S}^{*}(\alpha)=\mathcal{C S}_{1}^{*}(\alpha, 1)$ obtained by Ratti [9]. Putting, moreover, $\alpha=0$ we get the radius of starlikeness of the class $\mathcal{C S}^{*}=\mathcal{C S}_{1}^{*}(0,1)$ obtained by MacGregor [10]. 
Authors' contributions

All authors jointly worked on the results and they read and approved the final manuscript.

\section{Author details}

'Department of Mathematics, Abdul Wali Khan University, Mardan, Pakistan. ${ }^{2}$ Faculty of Mathematics and Natural Sciences, University of Rzeszów, Rzeszów, 35-310, Poland. ${ }^{3}$ Department of Mathematics, GC University Faisalabad, Faisalabad, Pakistan. ${ }^{4}$ Department of Mathematics, Rzeszów University of Technology, Rzeszów, 35-959, Poland.

\section{Acknowledgements}

This work is partially supported by the Centre for Innovation and Transfer of Natural Sciences and Engineering Knowledge, University of Rzeszów.

Received: 11 October 2014 Accepted: 3 December 2014 Published: 06 Jan 2015

\section{References}

1. Silverman, H: Products of starlike and convex functions. Ann. Univ. Mariae Curie-Skłodowska, Sect. A 29, 109-116 (1975)

2. Dimkov, G: On products of starlike functions. I. Ann. Pol. Math. 55, 75-79 (1991)

3. Aleksandrov, IA: On the star-shaped character of the mappings of a domain by functions regular and univalent in the circle. Izv. Vysš. Učebn. Zaved., Mat. 4(11), 9-15 (1959)

4. Stankiewicz, J, Świtoniak, B: Generalized problems of convexity and starlikeness. In: Complex Analysis and Applications '85 (Varna, 1985), pp. 670-675. Publ. House Bulgar. Acad. Sci., Sofia (1986)

5. Świtoniak, B: On a starlikeness problem in the class of functions $S_{\alpha}^{*}$. Folia Sci. Univ. Tech. Resov. 14, 17-27 (1984)

6. Dimkov, G, Dziok, J: Generalized problem of starlikeness for products of $p$-valent starlike functions. Serdica Math. J. 24, 339-344 (1998)

7. Dziok, J: Generalized problem of starlikeness for products of close-to-star functions. Ann. Pol. Math. 107, 109-121 (2013)

8. Nunokawa, M, Causey, WM: On certain analytic functions bounded argument. Sci. Rep. Fac. Educ., Gunma Univ. 34, 1-3 (1985)

9. Ratti, JS: The radius of univalence of certain analytic functions. Math. Z. 107, 241-248 (1968)

10. MacGregor, TH: The radius of univalence of certain analytic functions. Proc. Am. Math. Soc. 14, 514-520 (1963)

10.1186/1029-242X-2015-5

Cite this article as: Arif et al.: On products of multivalent close-to-star functions. Journal of Inequalities and Applications 2015, 2015:5

\section{Submit your manuscript to a SpringerOpen ${ }^{\circ}$ journal and benefit from:}

- Convenient online submission

Rigorous peer review

- Immediate publication on acceptance

- Open access: articles freely available online

- High visibility within the field

- Retaining the copyright to your article 